\section{Pesquisa sobre o aborto no Brasil: avanços e desafios para o campo da saúde coletiva}

\author{
Research on abortion in Brazil: gaps and \\ challenges for the public health field
}

\author{
1 Instituto de Saúde Coletiva, \\ Universidade Federal da \\ Bahia, Salvador, Brasil. \\ Correspondência \\ G. Menezes \\ Instituto de Saúde Coletiva \\ Universidade Federal da \\ Bahia. \\ Rua Basílio da Gama, s/n \\ Campus Universitário do \\ Canela, Salvador, BA \\ 40110-040, Brasil. \\ greice@ufba.br
}

\begin{abstract}
This paper provides a review of abortion studies produced in the field of public health in Brazil, highlighting current research gaps and challenges. Most studies focus on women admitted to public hospitals for treatment of incomplete abortion, so their scope is limited to abortions presenting complications. Women's profiles, abortion methods, motives, and immediate consequences for women's physical health are also included. However, there remains a need for studies on the following aspects: measuring abortion incidence; investigating cases of post-abortion complications and death; analyzing the relationship between abortion and contraception; investigating the impact of abortion on women's mental health; and incorporating men's perspectives. There is an urgent need for evaluative research on abortion care in public services. Research results should be disseminated widely, so as to help overcome any ideological bias in the current debate on abortion rights in the country.
\end{abstract}

Incomplete Abortion; Health Services Evaluation; Women's Health
Greice Menezes 1

Estela M. L. Aquino 1

Nas últimas três décadas, emergiu e se consolidou, no Brasil, um novo campo de produção científica articulando as temáticas de gênero, sexualidade e saúde reprodutiva 1 . Dentre os objetos que vêm sendo investigados nesse campo, o aborto tem sido estudado, reconhecendo-se sua importância como problema de saúde pública no país, buscando-se um diálogo com movimentos sociais nacionais e internacionais que incluem o tema como prioridade em suas agendas.

$\mathrm{O}$ estudo sobre o aborto tem o potencial de articular questões centrais e relevantes ao campo da saúde reprodutiva, desde as relações de gênero e os processos de decisão na esfera reprodutiva até a provisão de assistência e a garantia dos direitos sexuais e reprodutivos.

Na última década, coletâneas publicadas no país sobre saúde reprodutiva incluem a discussão sobre o aborto 2,3 e publicações inteiras foram dedicadas ao tema 4,5,6.

Recente e ampla revisão sobre vinte anos de pesquisa acerca do aborto no país aponta que na saúde coletiva esta produção, comparativamente a outras áreas, é oriunda majoritariamente de pesquisas empíricas. Esse acervo conta com a participação autoral importante de pesquisadoras de disciplinas como medicina - particularmente da gineco-obstetrícia - e enfermagem, cuja garantia de sigilo profissional viabilizou a realização de estudos em contextos hospitalares da rede pública de saúde, superando os limites impostos pela ilegalidade da prática do aborto 6 . 
A maioria das investigações foi realizada na Região Sudeste, sendo raras no Centro-Oeste e inexistentes no Norte. Há uma tradição de estudos em alguns grupos de pesquisa, chamando atenção que parte da produção foi elaborada sob a forma de dissertações e teses acadêmicas ${ }^{6}$.

O presente texto apresenta um panorama dos estudos sobre o aborto inseguro no país, no campo da Saúde Coletiva, buscando apontar lacunas nessa produção e desafios para a investigação do tema.

Para sua elaboração, procedeu-se a uma revisão da literatura nacional com a identificação de estudos empíricos, publicados em quatro periódicos - Cadernos de Saúde Pública, Ciência \& Saúde Coletiva, Revista Brasileira de Epidemiologia e Revista de Saúde Pública. Foram utilizados os termos "aborto", "aborto induzido", "aborto ilegal”, "aborto inseguro", "aborto hospitalar", "aborto provocado" e "abortamento" no campo de assunto. Foram excluídas aquelas referências em que a discussão relacionava-se aos casos de abortos previstos em lei, ou à interrupção da gravidez de fetos anencefálicos e a temas correlatos, como a contracepção de emergência; não foram examinados artigos que tratavam do debate jurídico e político sobre o aborto no país.

A análise do material foi conduzida tendo por base os seguintes eixos: magnitude do aborto no Brasil, repercussões do aborto sobre a saúde, juventude e vulnerabilidade ao aborto, aborto e contracepção, decisão pelo aborto e a perspectiva masculina frente ao aborto. Seu aprofundamento orientou a incorporação de referências complementares que constavam da bibliografia consultada.

\section{A magnitude do aborto no Brasil}

O aborto envolve aspectos de cunho moral e religioso, sendo objeto de forte sanção social. Essa condição implica dificuldades no seu relato pelas mulheres, particularmente em contextos de ilegalidade, como no Brasil. A investigação do aborto nessa situação requer cuidados metodológicos específicos, com implicações éticas no manejo do tema.

\section{Os estudos de base populacional}

No Brasil, são poucos os estudos de base populacional sobre o tema envolvendo amostras representativas de mulheres. Destacam-se algumas investigações locais 7,8,9,10,11, um estudo com escolares 12 e uma pesquisa multicêntrica, restrita à população de jovens 13 . As pesquisas Bem-Estar Familiar no Brasil/Demografhic Health Survey
(BEMFAM/DHS) de 1986 e 1996 14,15, apesar de incluírem questões sobre aborto, não divulgaram esses resultados nas respectivas publicações.

$\mathrm{Na}$ cidade do Rio de Janeiro, em inquérito domiciliar, Martins et al. 7 entrevistaram 1.784 mulheres de 15-49 anos, residentes em áreas de baixa renda. Aproximadamente um terço delas relatou um aborto, sendo $16,9 \%$ provocado; $23,6 \%$ das gestações declaradas terminaram em aborto.

As pesquisas que utilizaram a Técnica de Resposta ao Azar (TRA) alcançaram das mulheres relato mais freqüente de realização do aborto pela garantia da confidencialidade da informação no momento da entrevista. Silva 8, em 1987, e Silva et al. 16 em 1993, entrevistando mulheres em idade reprodutiva, residentes na zona urbana de São Paulo, constataram que, ao serem inquiridas diretamente, a maioria delas $(80 \%$ e $88 \%$, respectivamente) omitiu ter realizado um aborto, principalmente as mais jovens, as não-casadas, aquelas com um número de filhos nascidos vivos menor ou inferior ao considerado ideal, as não-usuárias de contraceptivos e aquelas com restrições à prática de aborto.

Em Pelotas, Rio Grande do Sul, Brasil, Olinto \& Moreira Filho 9,10 compararam o método da Urna com aquele das Questões Indiretas, em inquérito com 3.002 mulheres de 15-49 anos. No primeiro, a entrevistada depositava, em urna, sua resposta à questão direta se já havia feito "um aborto de um filho", enquanto que no segundo, oralmente, respondia a questões feitas por entrevistadores sobre se já havia engravidado em condições em que não podia ou não queria, e o desfecho desta gravidez. Os autores obtiveram relato de aborto induzido de 3,8\% aplicando as questões indiretas, elevando-se para $7,2 \%$ com o método da Urna. Eles consideram que mudanças na formulação da questão direta, que faz menção ao aborto "de um filho", poderiam contribuir para uma declaração ainda maior com o método da Urna.

Outros autores buscaram enfocar a própria interpretação das mulheres sobre a experiência de aborto. Pesquisas realizadas em São Paulo 11, Fortaleza (Ceará) 17 e Porto Alegre (Rio Grande do Sul) 18 constataram que elas tendem a negar a prática. Entretanto, declaram mais facilmente aquilo que consideram procedimentos de regulação menstrual (uso de chás, beberagens com ou sem drogas abortivas), em situações de gravidez presumida ou mesmo confirmada.

Para Nations et al. 17, mais do que manipulações conscientes de negação, as práticas declaradas pelas mulheres, entendidas como procedimentos de regulação menstrual, constituem estratégias de controle de sua fecundidade, em 
contextos restritivos e punitivos da realização do aborto. Já Leal \& Lewgoy 18 discutem como uma gravidez não-legitimada socialmente (pelo parceiro, pela família de ambos, pela rede social) abre a possibilidade de sua interrupção, com recurso a métodos não identificados como abortivos, mas para “fazer baixar as regras", integrando, portanto, uma rotina contraceptiva.

A realização de estudos multicêntricos propicia a comparação de realidades distintas em um país de contrastes socioculturais como o Brasil. Um caso exemplar é a Pesquisa GRAVAD - Gravidez na Adolescência: Estudo Multicêntrico sobre Jovens, Sexualidade e Reprodução no Brasil, com amostra representativa de 4.634 moças e rapazes de 18-24 anos de Salvador (Bahia), Rio de Janeiro e Porto Alegre. Nesse inquérito domiciliar, a menção ao aborto foi inicialmente obtida como alternativa de resposta ao desfecho de eventual gravidez em cada um dos relacionamentos que integravam a trajetória afetivo-sexual dos jovens; em seguida, em um bloco específico sobre gravidez, sumarizando as informações sobre aborto, o que permitia checar inconsistências entre as respostas. Embora não se possa afastar a possibilidade de sub-relato, 7,5\% delas e 12,4\% deles, ao falarem das parceiras, relataram a experiência de aborto provocado na adolescência. Esses resultados convergem com os de outras pesquisas, evidenciando maior magnitude do aborto, em ambos os sexos, entre jovens baianos, e menor entre gaúchos, com os cariocas em posição intermediária 13 .

As informações provenientes de inquéritos domiciliares, ainda que passíveis de subdeclaração, permitem investigar as características dos indivíduos, de sua família e o contexto do aborto, o que é difícil de alcançar por meio de outras fontes, além de possibilitar o contraste entre subgrupos que interromperam ou não a gravidez.

\section{Estimativas a partir de internações} hospitalares

Nos anos 1990, pesquisa publicada pelo Instituto Alan Guttmacher (AGI), desenvolvida no Brasil, Chile, México, Peru, Colômbia e República Dominicana, com base em registros de curetagens realizadas nos serviços públicos e em entrevistas com profissionais de saúde, estimou, para 1991, a ocorrência no Brasil de 1.443.350 abortos. Isto expressava uma taxa de 3,7 abortos/ 100 mulheres de 15-49 anos, com $31 \%$ das gravidezes terminando em aborto 19 .

Singh \& Sedgh 20, analisando a influência do aborto na determinação dos níveis e da tendência da fecundidade na Colômbia, México e Brasil, entre os anos 1970 e início da década de noventa, discutem que, ao final do período, o Brasil apresentava uma taxa de fecundidade geral $13 \%$ mais baixa do que a esperada, em função do aumento contínuo de abortos, desde os anos 1970. Para essas autoras, como já havia sido assinalado por Barbosa \& Arilha 21, a difusão do uso do Misoprostol, apesar da sua proibição, facilitou o recurso ao aborto, ao ser vendido ilegalmente no país, em uma realidade que impunha às mulheres obstáculos ao acesso a contraceptivos, mesmo com aumento da esterilização feminina.

Trabalhos publicados nos anos 1990 enfocaram a singularidade da experiência das brasileiras com o Misoprostol. Parte dessa produção foi publicada em periódicos estrangeiros, chamando atenção da associação do uso do Misoprostol com malformações fetais, em casos de tentativas não-exitosas de aborto 22,23,24.

Para o período 1994/1996, os dados do AGI foram atualizados por Corrêa \& Freitas 25 , registrando um decréscimo, mas estimando valores entre 728.100 a 1.039 .000 abortos provocados (médio e máximo, respectivamente).

Mais recentemente, Monteiro \& Adesse 26,27 estimaram a ocorrência do aborto no país, com base nas hospitalizações do Sistema de Informações Hospitalares do Sistema Único de Saúde (SIH/SUS). Em série histórica de 1992 a 2005, confirmam, para todas as regiões e grupos etários, tendência de redução das internações por abortamento de 1991 a 1996 e estabilização até 2005. Nesse último ano, estimou-se a realização de 1.054.242 abortos, com taxa média de 2,07 abortos / 100 mulheres entre 15-49 anos, mais elevada que em países da Europa Ocidental (1,1 aborto/100 mulheres de 15-44 anos), onde há acesso fácil aos contraceptivos e o aborto é legal 28.

\section{Repercussões do aborto sobre a saúde}

\section{Mortalidade: a face mais grave do problema}

Desde os anos 1980, registra-se em pesquisas a subnotificação das mortes por aborto, apontadas como as mais malnotificadas dentre as mortes maternas ${ }^{29}$. Embora com tendência de redução no Brasil 30 , figuram como a terceira causa nesse grupo, em decorrência de complicações infecciosas ou hemorrágicas 31 .

A única pesquisa de abrangência nacional, realizada nas capitais brasileiras e no Distrito Federal em 2002 32, evidenciou que 11,4\% dos óbitos maternos foram devidos a complicações de abortos. Entretanto, proporções mais elevadas foram registradas em investigações locais, destacando-se Recife (Pernambuco) - onde, em meados dos anos 1990, o aborto representava 
uma das primeiras causas desses óbitos 29 - e Salvador - onde o aborto manteve-se em 199333 e em 199834 como a principal causa isolada, responsável respectivamente por $36,4 \%$ e $22,5 \%$ dos óbitos maternos. Recentemente, em Porto Alegre, constatou-se que infecções por aborto, juntamente com distúrbios hipertensivos da gravidez, foram as principais causas de morte materna, cada uma com $15 \%$ dos óbitos 35 .

As mortes por aborto atingem preferencialmente mulheres jovens, de estratos sociais desfavorecidos, residentes em áreas periféricas das cidades. São também mais acometidas as negras que apresentam um risco três vezes superior de morrer por essa causa 27,36 , quando comparadas às brancas.

Um aspecto a ser elucidado relaciona-se ao achado recorrente em estudos que investigam óbitos maternos de jovens grávidas por suicídio. Nas entrevistas com familiares, a descoberta da gravidez e a tentativa de aborto situam-se no centro das circunstâncias que levaram à sua morte, o que instiga investigações sobre essa articulação 33,34.

\section{Complicações do aborto e assistência hospitalar}

Ao longo da última década, a curetagem pósaborto tem sido um dos procedimentos obstétricos mais realizados em hospitais públicos 31,37 . Com diferenças regionais importantes, as internações por abortamento são mais freqüentes no Nordeste e Sudeste do país, observandose, entretanto, nessas regiões, assim como no Sul, em todas as faixas etárias, uma redução de sua ocorrência entre 1992 e 2005. Diferentemente, no Centro-Oeste, as hospitalizações mantiveram-se praticamente inalteradas, com leve aumento entre mulheres de 25 a 49 anos e, no Norte, elevaram-se entre aquelas com menos de 40 anos 26 .

Estudos que enfocam as complicações pósaborto destacam a menor ocorrência de eventos infecciosos e hemorrágicos com o uso do Misoprostol 38,39, situação distinta daquela identificada em pesquisas realizadas no início dos anos 80, em que métodos mais agressivos para interrupção da gravidez eram citados.

Não foram localizados estudos sobre morbidade grave devido a complicações do aborto. $\mathrm{Na}$ literatura internacional, têm proliferado investigações sobre casos de mulheres que desenvolveram condições mórbidas graves por causas maternas, mas que sobreviveram - em inglês, casos denominados near miss. Trabalhos identificados no Brasil assinalam a importância dessa vertente de análise, obtendo dados mais acurados ao checar a informação diretamente das mulheres, além de permitir a avaliação da qualidade da assistência obstétrica 40 .

Poucos estudos foram desenvolvidos sobre a atenção prestada às mulheres que abortam. São de natureza qualitativa, realizados na sua maioria em serviços públicos, por enfermeiras diretamente envolvidas no cuidado às mulheres. Evidenciam como a atenção está centrada em cuidados corporais, muitas vezes de modo técnico e impessoal, com pouca escuta e atenção às necessidades das mulheres 41,42 . Nas maternidades, identificam-se espaços mínimos para atendimento, com pouca privacidade. Em várias ocasiões, elas esperam longamente a realização da curetagem e raras vezes lhes são fornecidas explicações sobre os procedimentos realizados ou os cuidados requeridos pós-procedimento, inclusive a contracepção posterior 41,42,43.

Em estudo etnográfico realizado em uma maternidade pública de Salvador, ficou claro como este tipo de unidade é pensado exclusivamente como local destinado a parturientes. Para além dos maus-tratos na relação profissional/usuária, discute-se como a discriminação é também simbólica e está institucionalizada na estrutura física, nos espaços destinados às mulheres que abortam e na forma de organização do atendimento 43 .

Outro aspecto pouco estudado refere-se aos custos da atenção ao aborto. O grande volume de internações para tratamento de complicações e eventuais seqüelas pós-aborto têm implicado gastos elevados para o setor saúde. Em um dos poucos trabalhos que menciona essa dimensão, Costa 38 avalia que, em 1991, no Rio de Janeiro, o total gasto com internações por essas causas seria suficiente para que o estado assumisse a realização de aproximadamente 62 mil abortos seguros, ou seja, $91 \%$ dos procedimentos estimados para aquele ano.

\section{Aborto e saúde mental}

São escassos os estudos no país sobre as repercussões do aborto na saúde mental das mulheres; os que foram localizados analisam a reação delas logo após a realização da interrupção ou em períodos próximos. De forma convergente, registram relatos ambíguos das mulheres, conjugando sentimentos de mal-estar físico ou emocional com alívio e bem-estar 44,45.

Deve ser mencionado o trabalho de Bailey et al. 46, exemplo raro de estudo prospectivo, comparando 196 adolescentes internadas com aborto incompleto (sendo $66,7 \%$ provocado) com 367 jovens no pré-natal. Se no período inicial, as primeiras apresentaram o menor escore de auto-estima, após um ano este padrão se inver- 
teu, com maior elevação da auto-estima entre as que abortaram, comparado com as que levaram a gravidez adiante.

As repercussões psíquicas do aborto constituem aspecto polêmico na literatura internacional. A comparação entre os estudos é difícil pelas diferenças amostrais, diversidade das medidas da saúde mental e dos períodos de realização das entrevistas em relação ao momento do aborto. Limites metodológicos são apontados, como ausência de grupos de comparação e não ajuste de fatores confundidores. Em estudos longitudinais, destacam-se o pouco tempo de seguimento dos grupos, as perdas importantes de acompanhamento e a falta de informação sobre não-participantes 47 .

Em outros países não há evidências de efeitos importantes do aborto sobre a saúde mental do ponto de vista populacional 48 . Onde sua prática é crime, como no Brasil, a investigação das repercussões psíquicas do aborto merece particular atenção. Para muitas mulheres, o longo percurso até a obtenção dos meios para abortar, a falta de atenção humanizada nos serviços de saúde, a divulgação da prisão de pacientes quando ainda internadas tornam dramáticas suas vivências, merecendo estudos que enfoquem a violência institucional e suas repercussões sobre a saúde dessas mulheres.

\section{Juventude e vulnerabilidade ao aborto}

O perfil das mulheres que recorrem ao aborto jovens, não-unidas, com pouca escolaridade, estudantes ou trabalhadoras domésticas - é registrado em pesquisas realizadas em hospitais brasileiros 49,50,51,52. Apesar da regularidade dos achados, apontando a vulnerabilidade das jovens, ao se enfocar o fenômeno neste segmento populacional constata-se que o aborto não ocorre de forma homogênea.

Entre universitárias paulistas, em sua primeira gestação, metade optou por um aborto 53 . Isso foi confirmado na Pesquisa GRAVAD, verificando-se que moças com renda familiar per capita e escolaridade mais elevadas relataram ter abortado, respectivamente, 4,6 e 3,8 vezes mais que aquelas mais pobres e menos escolarizadas. Assim, embora a gravidez tenha sido mais rara entre jovens de estratos sociais mais favorecidos, terminava mais freqüentemente em aborto. Chama atenção a situação das moças provenientes de famílias de renda mais baixa, que alcançaram uma escolaridade mais elevada do que suas mães: tal como as jovens mais privilegiadas relataram menos gravidez, mas se isto ocorreu, recorreram mais ao aborto. Mas ao efetivarem a prática, não o fizeram nas mesmas condições que as primeiras, tendo usado menos clínicas ou consultórios privados, mais tardiamente e por meio de técnicas menos seguras 54 .

$\mathrm{O}$ recurso ao aborto pelas jovens reforça ainda mais a argumentação de que as gestações que o originaram são consideradas sempre "não-desejadas". Entretanto, o aborto na juventude deve ser analisado em uma perspectiva mais ampla, à luz das mudanças ocorridas nas sociedades ocidentais, com a massificação da escolarização feminina, a disseminação dos valores do feminismo e o recurso a contraceptivos eficazes. Nesse cenário, as normas sociais relativas à reprodução, ao estabelecerem como ideal um número reduzido de filhos e definirem o momento propício para tê-los, qualificam a gravidez na juventude - e particularmente na adolescência - como despropósito, configurando-se como obstáculo às aspirações escolares e profissionais, sobretudo para aquelas que detêm maior capital sócio-cultural 13. Adicionalmente, a ausência de autonomia material e financeira dos jovens e o engajamento em relações ainda não consolidadas contrariam a representação dominante que valoriza a chegada do filho sob certas condições 55 .

\section{Aborto e contracepção}

Investigações nacionais realizadas com mulheres em maternidades públicas evidenciam que a maior parcela delas conhece os contraceptivos, sobretudo pílula e preservativo, embora o conhecimento sobre concepção e contracepção seja frágil, inconsistente, explicando em parte o uso pouco efetivo dos contraceptivos 56,57.

Outros estudos, entretanto, discutem que, independentemente da qualidade dessa informação, problemas de acesso aos contraceptivos - poucos métodos disponíveis, descontinuidade de suprimento, insuficiência de locais organizados para atendimento das demandas - se colocam como barreiras para grande parcela das mulheres que utiliza os serviços públicos 58,59.

Esses aspectos, todavia, não expressam a complexa relação das mulheres com a contracepção. Outros determinantes mediatos modelam as práticas contraceptivas. Essas práticas se situam em meio a distintas lógicas, devendo-se levar em consideração as relações de gênero aí implicadas, e a necessidade da prescrição médica para obtenção de alguns métodos. As situações de subordinação das mulheres se refletem no escasso diálogo com seus companheiros, na reticência ou mesmo na recusa destes em usar certos métodos. Embora a contracepção continue como um domínio feminino, a escolha do 
método pode ser feita em função de preferências dos homens; ainda é possível que, mesmo sem consultá-los, as mulheres antecipem as reações destes, ao interiorizarem sua responsabilidade em evitar a gravidez, ou em situações em que não tenham confiança na atitude do parceiro ${ }^{55}$.

Em tempos de AIDS, deve-se articular a necessidade de evitar uma gravidez e de se proteger contra a infecção pelo HIV. Trabalhos sinalizam que a segunda é secundarizada quando se está diante de um parceiro no qual se confia $60 \mathrm{ou}$ quando a relação se estabiliza, levando em ambas as situações ao abandono do preservativo.

Embora a contracepção seja uma das ações previstas nas políticas de saúde desde a década de 1980, a implementação heterogênea das ações no país não assegura a sua efetividade. Com relação à contracepção da emergência, a despeito de ser objeto de norma ministerial e do reconhecimento da sua eficácia pelo Conselho Federal de Medicina, para evitar uma gravidez após relações sexuais desprotegidas é pequena sua divulgação e prescrição nos serviços de saúde. Quanto à esterilização voluntária, mesmo após sua regulamentação pela Portaria $n^{\circ} .144$ do Ministério da Saúde, persistem obstáculos ao acesso 61 .

Trata-se, portanto, de um cenário desfavorável para as mulheres, particularmente as mais jovens, pois diante de uma relação sexual desprotegida só lhes resta o recurso ao aborto quando uma gravidez não-prevista ocorre.

\section{A decisão pelo aborto na perspectiva das mulheres}

No campo da Saúde Coletiva, são raras as investigações específicas sobre o processo de decisão de um aborto. No âmbito dessa discussão torna-se necessário distinguir os motivos alegados para a decisão - o "porquê" - do contexto e das circunstâncias em que esta se constrói - o "como" - embora assinalando a artificialidade desta distinção entre elementos estreitamente relacionados 62

Os motivos relatados para a interrupção da gravidez são variados segundo a idade, situação conjugal e contexto social. Entre aquelas sem filhos, uma alegação freqüente é a necessidade de postergar a maternidade para possibilitar a continuação de estudos ou trabalho 13. A falta de recursos financeiros é apontada, sobretudo, quando se investigam mulheres de camadas populares 7,38 , não sendo tão relevante naquelas de estratos sociais favorecidos 44,53.

A natureza e o grau de legitimidade da relação com o parceiro são aspectos relevantes para jus- tificar um aborto. A despeito da multiplicidade dos arranjos familiares da atualidade, socialmente o projeto de um filho exige um casal engajado em uma relação estável 55 .

A explicação da decisão de abortar privilegia algumas razões em detrimento de outras. A necessidade de legitimação do aborto é feita como uma justificação, isto é, orientada para uma esfera moral, estabelecendo esta escolha como "um mal menor". Trata-se de justificativas que remetem também ao filho, tendo a decisão sido tomada em seu benefício, pela impossibilidade de lhe garantir uma vida digna 63 .

Sobre o processo da decisão em si, os escassos estudos sobre esta dimensão no país têm sido conduzidos, sobretudo, nas disciplinas das ciências humanas, mediante pesquisas qualitativas 64,65 , considerando-se a dificuldade de abordar uma questão eminentemente subjetiva em estudos quantitativos. A construção da decisão deve ser compreendida como um processo, comportando idas e vindas, numa acumulação de fatores que podem culminar no aborto 55 . Nesse sentido, Peres \& Heilborn 65 , contrapondo-se a uma visão reducionista da decisão frente ao aborto, identificam um continuum entre não ter pensado na possibilidade do aborto, sua cogitação, tentativa e efetivação.

A decisão costuma ser atribuída unicamente às mulheres, negligenciando-se a influência de outros atores, variável segundo as fases do ciclo de vida destas. A atitude inicial das mulheres, apesar de crucial na decisão, está sujeita a modificações em função de projetos pessoais, das circunstâncias do momento, das reações do parceiro e das redes familiar e social 55 .

\section{A perspectiva masculina frente ao aborto}

A incorporação dos homens nas pesquisas sobre gravidez, contracepção e aborto tem permitido perceber como se elaboram os processos de negociação e decisão no campo da reprodução, este compreendido como espaço de relação 66,67,68.

A externalidade da gravidez ao corpo masculino, ao permitir aos homens a alternativa de se excluir fisicamente da decisão e das conseqüências desta, faz supor que esta exclusão é total, sobretudo quando o resultado é sua interrupção. O tipo de vínculo com a parceira parece determinante para se assumir a gravidez e o filho, sendo fundamental o reconhecimento da relação, com o casal constituído, ou em vias de fazê-lo. Nos casos em que a gravidez não é aceita, a ilegitimidade do vínculo é alegada, comportando inclusive suspeição acerca da paternidade 69 . 
O afastamento dos homens jamais é completo; de alguma forma influem e interagem nesse processo. A participação deles pode se dar pela busca de informação sobre meios e locais para realização do procedimento, pelo pagamento deste, pela presença junto à parceira no momento do abortamento e/ou oferecendo-lhe apoio e suporte emocional 64 .

A inclusão dos homens nas pesquisas traz perspectiva complementar à das mulheres, habitualmente únicas instadas a se pronunciar sobre o tema. Na Pesquisa GRAVAD, destaca-se maior relato de aborto pelos rapazes do que pelas moças entrevistadas 54 , diferentemente de Pirotta \& Schor 70 , que constatam proporções semelhantes de relato entre universitários paulistas.

As declarações de aborto feitas pelos rapazes devem ser interpretadas com cautela, já que os modelos para compreensão do fenômeno entre eles são menos conhecidos e mais complexos. Um maior relato de aborto (da parceira) entre homens pode indicar menor dificuldade em declará-lo, pela externalidade da gravidez, mas também pelo reforço simbólico da sua própria fertilidade e masculinidade. Pode igualmente expressar imprecisões na informação sobre gestações das parceiras. Não se pode afastar que o maior relato de aborto entre eles decorra de diferenças de gênero nas trajetórias afetivo-sexuais. As experiências masculinas comportam uma iniciação sexual mais cedo, um maior número de parceiras eventuais, muitas vezes simultâneas, implicando maior risco de gravidez ${ }^{71}$.

\section{Lacunas e desafios para investigação sobre aborto}

Nas últimas décadas, o cenário social e político brasileiro apresenta mudanças e permanências quanto ao aborto. Persiste a não-flexibilização da legalização punitiva em vigor, a influência de grupos religiosos contrários à descriminalização da prática, com relações orgânicas no âmbito das câmaras legislativas estaduais e federal, embora tenha havido maior visibilidade social do tema e atuação mais organizada de articulações feministas pró-descriminalização e legalização.

Desde a década passada, tem-se generalizado entre distintos segmentos da população a percepção de um ideal de prole reduzida. Nessa perspectiva, se persistirem as dificuldades de acesso a um leque mais diversificado de contraceptivos reversíveis - no país o uso é concentrado na pílula hormonal e no preservativo masculino -, as mulheres continuarão sendo levadas a recorrer a medidas radicais, como a esterilização feminina e o aborto 72 .
A discussão do aborto não pode desconhecer a situação brasileira de desigualdade e exclusão social de parcela da população, cujos efeitos atingem particularmente mulheres e jovens. Essa heterogeneidade social conforma distintas possibilidades para indivíduos de diferentes estratos, com diversas configurações de família, redes de sociabilidade, condições materiais de existência, percursos escolares, perspectivas e inserções no mercado de trabalho. Nessas trajetórias, os projetos de maternidade/paternidade assumem lugares e significados específicos. Diante de uma gravidez não-prevista, esses determinantes implicarão, para eles e elas, possibilidades diversas frente à decisão de sua interrupção.

No Brasil, o aborto configura-se em problema de saúde pública. A investigação das mortes por aborto tem permitido, a despeito do pequeno número absoluto de eventos, demonstrar o efeito da legislação, que mantém elevado o risco de morte de mulheres que abortam em condições inseguras. Os estudos são inúmeros, embora com comparabilidade limitada pela diversidade da metodologia empregada, pelas diferenças da cobertura do sistema de mortalidade e da situação de implantação da vigilância ao óbito materno universo de óbitos investigados, funcionamento dos Comitês de Morte Materna e incorporação das suas análises ao sistema. Deve-se investir na investigação das especificidades dos óbitos por aborto, na identificação dos casos de morbidade grave (ou near miss) e os fatores associados. A relação entre gravidez, aborto e suicídio merece ser explorada.

A maioria das pesquisas está concentrada em populações específicas, sendo estas, em sua maioria, mulheres admitidas em hospitais públicos com aborto incompleto. Apresentam assim um viés de seleção, já que reportam apenas uma parcela dos abortos, aqueles com complicações que necessitam hospitalização.

Essas investigações são importantes para a descrição do perfil das mulheres que informaram provocar um aborto, os métodos utilizados, as razões alegadas para fazê-lo, seu nível de informação e utilização de contraceptivos, e as conseqüências imediatas para a saúde física.

Entretanto, a análise efetuada por esses estudos, além de limitada a algumas variáveis, restringe-se a um enfoque transversal do problema, sem um modelo explicativo capaz de articular tais variáveis para apreensão da complexidade do fenômeno. Sexo e idade são, muitas vezes, analisados em uma perspectiva naturalizada, reduzidos à sua dimensão empírica e destituídos de seu significado social.

Assim, poucos estudos discutem os determinantes do aborto, de forma a analisar os fatores 
sociais implicados. Os relatos de aborto provocado são distintos, segundo gênero, raça e grupo social, refletindo a multiplicidade de significados que podem ser atribuídos a cada gravidez e ao próprio aborto, associados a fatores de diferentes ordens. Tal análise permitiria desvelar as desigualdades sociais de mulheres e homens frente ao risco de uma gravidez não-prevista, inserindo a discussão do tema nos marcos dos direitos sexuais e reprodutivos.

Pesquisas apontam maior vulnerabilidade de adolescentes e jovens, mas é necessário maior discussão sobre os determinantes e as experiências da gravidez e do aborto nessas etapas da vida.

As investigações ressaltam a carência de informação sobre contraceptivos por parte das mulheres, sobretudo das mais jovens; ou, mesmo, o achado supostamente paradoxal de que, apesar de conhecerem os métodos, não os utilizaram no período anterior ao episódio da gravidez estudada, sendo assim culpabilizadas por este comportamento "irracional". Essa forma de responsabilização individual no mínimo desconhece o contexto social das brasileiras e as dificuldades materiais que vivenciam.

Na medida em que parte significativa dos estudos foi realizada com populações de baixa renda, uma associação entre pobreza e aborto está presente, restringindo a diversidade de situações. As pesquisas realizadas fora dos serviços de saúde, com universitários de ambos os sexos 53 , alunas e funcionárias de universidades brasileiras 73 e aquelas de base populacional 11,12,13,14,15,16,17,18, evidenciaram a heterogeneidade das mulheres que recorrem ao aborto, sendo este evento parte das suas trajetórias reprodutivas.

Homens são raramente incluídos nas pesquisas. Alguns estudos apresentam informações indiretas fornecidas pelas mulheres entrevistadas 74,75 , com poucas investigações em que eles integram o universo empírico do estudo 53,54,76. Além disso, muitas pesquisas são de natureza qualitativa, sendo escassas as que permitem inferências populacionais.

Na produção do tema na área da Saúde Coletiva, aliada à perspectiva clínico-epidemiológica, é crescente a interlocução com outros campos do conhecimento. Um maior diálogo entre esses campos contribuirá para construir um arcabouço teórico, de modo a contextualizar e conferir sentido aos padrões epidemiológicos encontrados. Disciplinas das ciências humanas e sociais trazem novas questões de pesquisa, vislumbrando outros olhares acerca do assunto.

Após vinte anos de investigação sobre o tema, constata-se o aprofundamento de tendência registrada por Barroso 77, em artigo de revisão.
Ainda que não restrita a publicações da área da saúde, a autora destacava naquela ocasião mudanças na abordagem do assunto, com diversificação das questões, buscando-se uma compreensão mais objetiva dos fenômenos. Sobretudo, no início da década de 1990, novos aspectos relacionados ao aborto foram trazidos com a realização de pesquisas fora do ambiente hospitalar e a investigação de novas questões 78 .

Entretanto, permanecem alguns limites na produção científica, muito semelhantes àqueles apontados por investigadoras latino-americanas há dez anos 79, posteriormente pelas pesquisadoras Guillaume \& Lerner 80 , em amplo levantamento bibliográfico sobre o tema na América Latina e Caribe e, mais recentemente, na revisão de Diniz \& Correa 6 .

Um dos limites refere-se à necessidade de estudos comparativos entre as várias regiões do país. Para sua superação, recomenda-se a realização de pesquisas de caráter multicêntrico e base populacional, para mensuração da incidência do aborto, utilizando técnicas de validação da informação. A combinação de estratégias multidisciplinares favorecerá a investigação dos determinantes do aborto sob diferentes perspectivas. Nos estudos, é preciso não só incluir amostras de mulheres urbanas, mas também das residentes em áreas rurais e cidades menores, bem como populações excluídas, como indígenas, quilombolas e portadoras de deficiência.

Antigas temáticas, como a relação do aborto com anticoncepção, devem ser analisadas em novas perspectivas, permitindo reconstruir as trajetórias contraceptivas das mulheres, as variações da prática contraceptiva segundo distintos fatores, sobretudo quanto aos tipos de parceria e os processos de transição de um método a outro, momentos que favorecem falhas contraceptivas.

A incorporação da perspectiva masculina deve ser incentivada, não só para conferir maior visibilidade aos homens nas questões reprodutivas, mas para discuti-las à luz das relações de gênero. Análise dos desfechos da gravidez segundo tipo e natureza da parceria permitirá compreender melhor a decisão pelo aborto, o papel dos homens nesta decisão, e suas atitudes diante da gravidez. Também propiciarão explorar situações de concordância e discordância, repercussões da decisão sobre a relação do casal e sobre suas vidas. As situações de homogamia/heterogamia - etária, de cor/raça, de religião - devem ser também investigadas.

Estudos sobre repercussões do aborto na saúde mental das mulheres devem ser empreendidos, sobretudo para se investigar situações de violência institucional, face à ilegalidade do 
evento. Isso permitirá orientar as capacitações dos profissionais de saúde envolvidos na atenção às mulheres.

É urgente a avaliação da atenção ao aborto nos serviços públicos, considerando as condutas preconizadas na Atenção Humanizada ao Abortamento: Norma Técnica, publicada em 2005 pelo Ministério da Saúde 31. A prática cotidiana dos serviços incorpora ainda de forma incipiente essas condutas. Merecem análises acerca das razões da resistência institucional à implantação da técnica de aspiração manual a vácuo, a despeito das evidências científicas que atestam menor risco associado e menor custo desta técnica quando comparada à curetagem uterina 81 .

Os resultados dos estudos acadêmicos devem ser objeto de divulgação mais ampla na sociedade, de modo a superar a visão maniqueísta e ideologizada que marca a discussão do direito ao aborto no país.

Um primeiro passo é a ampliação da compreensão acerca da heterogeneidade do universo configurado pelas mulheres diante de uma gravidez. Essa heterogeneidade contrasta com a uniformidade com que são tratadas pelas políticas públicas e pelos profissionais, particularmente os de saúde 82 . É preciso incluir essa discussão na formulação das ações, na formação e educação continuada desses profissionais, de modo que sejam considerados aspectos relativos ao contexto social em que as mulheres estão inseridas, seu envolvimento com o parceiro da gravidez, o suporte da família e a rede social em que se incluem.

\section{Resumo}

O texto apresenta um panorama dos estudos sobre aborto no país, no campo da Saúde Coletiva, apontando lacunas e desafios para a investigação. A maioria das pesquisas está concentrada em hospitais públicos, com mulheres admitidas para tratamento do aborto incompleto, restringindo-se portanto aos abortos que apresentaram complicações. Descrevem o perfil das mulheres, métodos e razões para o aborto e conseqüências imediatas para a saúde física. Entretanto, permanecem limites relacionados à necessidade de estudos para mensuração da incidência do aborto; para investigação das especificidades dos óbitos por aborto
A diminuição da ocorrência dos abortos inseguros demanda ações que extrapolam o âmbito do setor saúde. Investimentos em educação são necessários. A maior escolaridade tem sido apontada nos estudos como um grande diferencial quanto aos comportamentos reprodutivos. Mesmo com a tendência observada no país de redução da idade de iniciação sexual das mulheres e conseqüente aproximação dos calendários feminino e masculino, o maior acesso à educação constitui ferramenta para que os jovens possam inclusive manejar a contracepção de forma mais adequada.

Educação sexual nas escolas, em uma perspectiva não-sexista e não-homofóbica, deve ser implementada. A despeito da inserção de conteúdos específicos nos Parâmetros Curriculares Nacionais, aprovados desde 1996, ainda é incipiente sua aplicabilidade, dada a necessidade de investimentos na formação de professores.

Ações para garantir às mulheres (e aos homens) informações e acesso aos contraceptivos, incluída a contracepção de emergência, devem ser realizadas no âmbito do SUS, na perspectiva da integralidade da atenção, como preconizado pelo Programa de Atenção Integral de Saúde da Mulher (PAISM) há duas décadas, e reiterado, mais recentemente, pela Política Nacional de Atenção Integral à Saúde da Mulher.

Por fim, reafirmando a laicidade do Estado brasileiro, a revisão da legislação do aborto vigente pode favorecer a implementação de ações que assegurem maior autonomia de mulheres e homens nas questões reprodutivas e lhes permitam vivenciar suas escolhas sem riscos à saúde. e casos de morbidade grave; para análise da relação do aborto com anticoncepção; para investigação das repercussões do aborto na saúde mental das mulheres e para incorporação da perspectiva masculina. É urgente o desenvolvimento de pesquisas de avaliação da atenção ao aborto nos serviços públicos. Os resultados dos estudos devem ser divulgados, contribuindo para superar a visão ideologizada da discussão do direito ao aborto no país.

Aborto Incompleto; Avaliação de Serviços de Saúde; Saúde da Mulher 


\section{Colaboradores}

G. Menezes elaborou o artigo. E. M. L. Aquino procedeu à revisão crítica do texto.

\section{Agradecimentos}

Este texto foi originalmente apresentado no seminário "Estudos sobre a Questão do Aborto em Países da América do Sul”, em comemoração aos 25 anos do Núcleo de Estudos de População da Universidade Estadual de Campinas (NEPO/UNICAMP). Registramos nosso agradecimento à Maria Isabel Baltar da Rocha (in memorian) pelo convite para o evento, cujos debates propiciaram o aprimoramento do trabalho, e a Tina Amado pela revisão cuidadosa do texto.

\section{Referências}

1. Aquino EML, Barbosa RM Heilborn ML, Berquó E. Gênero, sexualidade e saúde reprodutiva: a constituição de um novo campo na Saúde Coletiva. Cad Saúde Pública 2003; 19 Suppl 2:S198-9.

2. Giffin K, Costa SH, organizadores. Questões de saúde reprodutiva. Rio de Janeiro: Editora Fiocruz; 1999

3. Berquó E, organizador. Sexo \& vida: panorama da saúde reprodutiva no Brasil. Campinas: Editora Unicamp; 2003.

4. Faúndes A, Barzelatto J. O drama do aborto: em busca de um consenso. Campinas: Editora Komedi; 2004.

5. International Planned Parenthood Federation. Morte e negação: abortamento inseguro e pobreza. Rio de Janeiro: Bem-Estar Familiar no Brasil; 2007.

6. Departamento de Ciência e Tecnologia, Secretaria de Ciência, Tecnologia e Insumos Estratégicos. 20 anos de pesquisas sobre aborto no Brasil. Brasília: Ministério da Saúde; 2008. (Série B, Textos Básicos de Saúde).

7. Martins IR, Costa SH, Freitas SRS, Pinto CS. Aborto induzido em mulheres de baixa renda: dimensão de um problema. Cad Saúde Pública 1991; 7: 251-66.

8. Silva RS. O uso da técnica de resposta ao azar (TRA) na caracterização do aborto ilegal. Rev Bras Estud Popul 1993; 10:41-56.

9. Olinto MTA, Moreira Filho DC. Estimativa de aborto induzido: comparação entre duas metodologias. Rev Panam Salud Pública 2004; 15:331-6.

10. Olinto MTA, Moreira Filho DC. Fatores de risco e preditores para o aborto induzido: estudo de base populacional. Cad Saúde Pública 2006; 22:365-75.

11. Osis MJD, Hardy E, Faúndes A, Rodrigues T. Dificuldades para obter informações da população de mulheres sobre aborto ilegal. Rev Saúde Pública 1996; 30:444-51.
12. Castro MG, Abramovay M, Silva LB, organizadores Juventudes e sexualidade. Brasília: Organização das Nações Unidas para a Educação, a Ciência e a Cultura; 2004.

13. Heilborn ML, Aquino EML, Bozon M, Knauth DR, organizadores. O aprendizado da sexualidade: um estudo sobre reprodução e trajetórias sociais de jovens brasileiros. Rio de Janeiro: Editora Garamond; 2006.

14. Bem-Estar Familiar no Brasil. Pesquisa nacional sobre saúde materno-infantil e planejamento familiar. Rio de Janeiro: Bem-Estar Familiar no Brasil; 1986.

15. Bem-Estar Familiar no Brasil. Pesquisa nacional sobre saúde materno-infantil e planejamento familiar. Rio de Janeiro: Bem-Estar Familiar no Brasil; 1996.

16. Silva RS, Morell MGG. Em que medida as paulistanas recorrem ao aborto provocado. Rev Bras Estud Popul 2002; 19:249-61.

17. Nations MK, Misago C, Fonseca W, Correia LL, Campbell OM. Women's hidden transcripts about abortion in Brazil. Soc Sci Med 1997; 44:1833-45.

18. Leal OF, Lewgoy B. Aborto: uma contribuição antropológica à discussão. Revista de Filosofia Política 1998; 2:173-95

19. Alan Guttmacher Institute. Aborto clandestino: uma realidade latino-americana. New York: Alan Guttmacher Institute; 1994.

20. Singh S, Sedgh G. Relación del aborto con las tendencias anticonceptivas y de fecundidad en el Brasil, Colombia y México. Perspect Int Planif Famil 1997; n. espec.:2-13.

21. Barbosa RM, Arilha M. A experiência brasileira com o Cytotec. Revista Estudos Feministas 1993; 1:408-17.

22. Costa SH, Vessey MP. Misoprostol and illegal abortion in Rio de Janeiro, Brazil. Lancet 1993; 341:1258-61. 
23. Coêlho HL, Teixeira AC, Santos AP, Forte EB, Morais SM, La Vecchia C, et al. Misoprostol and illegal abortion in Fortaleza, Brazil. Lancet 1993; 341:1261-3.

24. Gonzalez CH, Marques-Dias MJ, Kim CA, Sugayama SM, Da Paz JA, Huson SM, et al. Congenital abnormalities in Brazilian children associated with misoprostol misuse in first trimester of pregnancy. Lancet 1998; 351:1624-7.

25. Corrêa S, Freitas A. Atualizando os dados sobre a interrupção voluntária da gravidez no Brasil. Revista Estudos Feministas 1997; 5:389-95.

26. Monteiro MFG, Adesse L. Estimativas de aborto induzido no Brasil e Grandes Regiões. In: Anais do $15^{\circ}$ Encontro Nacional de Estudos Populacionais. Belo Horizonte: Associação Brasileira de Empresas de Pesquisa; 2006. http://www.abep.nepo.uni camp.br/encontro2006/docspdf/ABEP2006_252. pdf. (acessado em 25/Jun/2007).

27. Monteiro MFG, Adesse L. Magnitude do aborto no Brasil: uma análise dos resultados de pesquisa. http://www.ims.uerj.br/downloads/ipas_ims_se minario_aborto.pdf (acessado em 25/Jun/2007).

28. Henshaw SH, Singh S, Haas T. La incidencia del aborto inducido a nivel mundial. Perspect Int Planif Famil 1999; n. espec:16-24.

29. Valongueiro S. Mortalidade (materna) por aborto: fontes, métodos e instrumentos de estimação. In: Anais do 12o Encontro Nacional de Estudos Populacionais. http://www.abep.nepo. unicamp.br/docs/anais/pdf/2000/Todos/Mor talidade\%20(Materna)\%20por\%20Aborto\%20 \%20Fontes,\%20Métodos....pdf. (acessado em 10/ Mar/2005).

30. Lima BGC. Mortalidade por causas relacionadas ao aborto no Brasil: declínio e desigualdades espaciais. Rev Panam Salud Pública 2000; 7:168-72.

31. Departamento de Ações Programáticas Estratégicas, Secretaria de Atenção à Saúde, Ministério da Saúde. Atenção humanizada ao abortamento: norma técnica. Brasília: Ministério da Saúde; 2005.

32. Laurenti R, Mello-Jorge MH, Gotlieb SLD. A mortalidade materna nas capitais brasileiras: algumas características e estimativa de um fator de ajuste. Rev Bras Epidemiol 2004; 7:449-60.

33. Compte GM. Mortalidade materna em Salvador, 1993 [Dissertação de Mestrado]. Salvador: Instituto de Saúde Coletiva, Universidade Federal da Bahia; 1995.

34. Menezes GMS, Aquino EML. Mortalidade materna na Bahia, 1998: relatório de pesquisa. Salvador: Instituto de Saúde Coletiva/Secretaria de Saúde do Estado da Bahia; 2001.

35. Riquinho DL, Correia SG. Mortalidade materna: perfil sócio-demográfico e causal. Rev Bras Enferm 2006; 59:303-7.

36. Martins AL. Mortalidade materna de mulheres negras. Cad Saúde Pública 2006; 22:2473-9.

37. Sorrentino SR, Lebrão ML. Os abortos no atendimento hospitalar do Estado de São Paulo, 1995. Rev Bras Epidemiol 1998; 1:256-67.

38. Costa SH. Aborto provocado: a dimensão do problema e a transformação da prática. In: Giffin K, Costa SH, organizadores. Questões de saúde reprodutiva. Rio de Janeiro: Editora Fiocruz; 1999. p. 163-86.
39. Hardy E, Alves G. Complicações pós-aborto provocado: fatores associados. Cad Saúde Pública 1992; 8:454-8.

40. Souza JPD, Cecatti JG, Parpinelli MA. Fatores associados à gravidade da morbidade materna na caracterização do near miss. Rev Bras Ginecol Obstet 2005; 27:197-203.

41. Motta IS. A relação interpessoal entre profissionais de saúde e a mulher em abortamento incompleto: o "olhar da mulher". Rev Bras Saúde Matern Infant 2005; 5:219-28.

42. Mariutti MG, Almeida AM, Panobianco MS. Nursing care according to women in abortion situations. Rev Latinoam Enferm 2007; 15:20-6.

43. McCallum CA, Reis AP, Menezes GMS. O atendimento ao aborto em uma unidade pública de saúde: as perspectivas de jovens usuárias e profissionais de saúde. In: Seminário Internacional Fazendo Gênero 7 [CD-ROM]. Florianópolis: Universidade Federal de Santa Catarina/Universidade Estadual de Santa Catarina; 2006.

44. Costa RG, Hardy E, Osis MJD, Faúndes A. A decisão de abortar: processos e sentimentos envolvidos. Cad Saúde Pública 1995; 11:97-105.

45. Pedrosa IL, Garcia TR. "Não vou esquecer nunca!”: a experiência feminina com o abortamento induzido. Rev Latinoam Enferm 2000; 8:50-8.

46. Bailey PE, Bruno ZV, Bezerra MF, Queiroz I, Oliveira $\mathrm{CM}$, Chen-Mok M. Adolescent pregnancy 1 year later: the effects of abortion vs. motherhood in Northeast Brazil. J Adolesc Health 2001; 29:223-32.

47. Adler NE, David HP, Major BN, Roth SH, Russo SH, Wyatt GE. Psychological responses after abortion. Science 1990; 248:41-4.

48. Cohen SA. Abortion and mental health: myths and realities. Guttmacher Policy Review 2006; 9:8-12.

49. Schor N. Investigação sobre a ocorrência de aborto entre pacientes de hospital de centro urbano do Estado de São Paulo, Brasil. Rev Saúde Pública 1990; 24:144-51.

50. Vianna AM, Anjos ACM, Costa CFF. Estudo do perfil epidemiológico das pacientes com aborto provocado na Maternidade da Encruzilhada. Rev IMIP 1990; 4:88-91.

51. Fonseca W, Misago C, Correia LL, Parente JAM, Oliveira FC. Determinantes do aborto provocado entre mulheres admitidas em hospitais em localidade da região Nordeste do Brasil. Rev Saúde Pública 1996; 30:13-8.

52. Fonseca W, Misago C, Freitas P, Santos E, Fernandes L, Correia L. Características sócio-demográficas, reprodutivas e médicas de mulheres admitidas por aborto em hospital da Região Sul do Brasil. Cad Saúde Pública 1998; 14:279-86.

53. Pirotta KCM, Schor N. Considerações sobre a interrupção voluntária da gravidez a partir do discurso de estudantes universitários da USP. In: Anais do Encontro Nacional de Estudos Populacionais. http://www.abep.nepo.unicamp.br/site_eventos_ abep/PDF/ABEP2004_430.pdf (acessado em 12/ Set/2005).

54. Menezes GMS, Aquino EML, Silva DO. Induced abortion during youth: social inequalities in the outcome of the first pregnancy. Cad Saúde Pública 2006; 22:1431-46. 
55. Bajos N, Ferrand M; l'Équipe GINE. De la contraception à l'avortement: sociologie des grossesses non prévues. Paris: Institut National de la Santé et de la Recherche Médicale; 2002.

56. Schor N, França AP, Siqueira AAF, Pirotta KCM, Alvarenga AA. Adolescência: vida sexual e anticoncepção. In: Anais do 11o Encontro Nacional de Estudos Populacionais. http://www.abep. nepo.unicamp.br/docs/anais/PDF/1998/a130.pdf (acessado em 20/Mai/2005).

57. Almeida MCC, Aquino EML, Gaffkin L, Magnani RJ. Uso de contracepção por adolescentes de escolas públicas na Bahia. Rev Saúde Pública 2003; 37:556-75.

58. Scavone L, Brétin H, Thébaud-Mony A. Contracepção, controle demográfico e desigualdades sociais: análise comparativa franco-brasileira. Revista Estudos Feministas 1994; 2:357-72.

59. Giffin K, Costa SH. As práticas contraceptivas e o aborto no Brasil. In: Family Health International, organizador. Reflexões sobre gênero e fecundidade no Brasil. São Paulo: Family Health International; 1995. p. 54-75.

60. Paiva V. Sexualidades adolescentes: escolaridade, gênero e o sujeito social. In: Parker R, Barbosa $\mathrm{RM}$, organizadores. Sexualidades brasileiras. Rio de Janeiro: Editora Relume-Dumará/Associação Brasileira Interdisciplinar de AIDS/Instituto de Medicina Social, Universidade do Estado do Rio de Janeiro; 1996. p. 213-34.

61. Berquó E, Cavenaghi S. Direitos reprodutivos de mulheres e homens face à nova legislação brasileira sobre esterilização voluntária. Cad Saúde Pública 2003; 19 Suppl 2:S441-53

62. Kero A, Jacobsson L, Högberg U, Lalos A. Legal abortion: a painful necessity. Soc Sci Med 2001; 53:1481-90.

63. Boltanski L. La condition fœtale: une sociologie de l'engendrement et de l'avortement. Paris: Gallimard; 2004

64. Ramirez-Galvez MC. Os impasses do corpo: ausências e preeminências de homens e mulheres no caso do aborto voluntário [Dissertação de Mestrado]. Campinas: Departamento de Antropologia Social, Universidade Estadual de Campinas; 1999.

65. Peres SO, Heilborn ML. Cogitação e prática do aborto entre jovens em contexto de interdição legal: o avesso da gravidez na adolescência. Cad Saúde Pública 2006; 22:1411-20.

66. Arilha M. Homens: entre a "zoeira" e a "responsabilidade". In: Arilha M, Ridenti SGU, Medrado B, organizadores. Homens e masculinidades: outras palavras. São Paulo: ECOS - Comunicação em Sexualidade/Editora 34; 1998. p. 51-78.

67. Arilha M. Homens, saúde reprodutiva e gênero: o desafio da inclusão. In: Giffin K, Costa SH, organizadores. Questões da saúde reprodutiva. Rio de Janeiro: Editora Fiocruz; 1999. p. 455-67.

68. Garcia SM. Conhecer os homens a partir do gênero e para além do gênero. In: Arilha M, Ridenti SGU, Medrado B, organizadores. Homens e masculinidades: outras palavras. São Paulo: ECOS - Comunicação em Sexualidade/Editora 34; 1998. p. 31-50.
69. Heilborn ML, Salem T, Rohden F, Brandão E, Knauth D, Victora C, et al. Aproximações sócio-antropológicas sobre a gravidez na adolescência. Horizontes Antropológicos 2002; 8:13-45.

70. Pirotta KCM, Schor N. Intenções reprodutivas e práticas de regulação da fecundidade entre universitários. Rev Saúde Pública 2002; 38:495-502.

71. Menezes GMS. Aborto, juventude e saúde: um estudo em três capitais brasileiras [Tese de Doutorado]. Salvador: Instituto de Saúde Coletiva, Universidade Federal da Bahia; 2006.

72. Ministério da Saúde. Pesquisa nacional de demografia e saúde da criança e da mulher 2006. Brasília: Ministério da Saúde; 2008.

73. Hardy E, Rebello I. Aborto entre alunas e funcionárias de uma universidade brasileira. Rev Saúde Pública 1993; 12:259-66.

74. Bruno ZV, Bailey PE. Gravidez em adolescentes no Ceará: maternidade ou aborto. In: Vieira EM, Fernandes MEL, Bailey PE, McKay A, organizadores. Seminário Gravidez na Adolescência. Rio de Janeiro: Associação Saúde da Família; 1998. p. 57-66.

75. Costa MCO, Lima IC, Martins Jr. DF, Santos CAST, Araújo FPO, Assis DR. Gravidez na adolescência e co-responsabilidade paterna: trajetória sociodemográfica e atitudes com a gestação e a criança. Ciênc Saúde Coletiva 2005; 10:719-27.

76. Duarte GA, Alvarenga AT, Osis MJD, Faúndes A, Hardy E. Perspectiva masculina acerca do aborto provocado. Rev Saúde Pública 2002; 36:271-7.

77. Barroso C. As pesquisas sobre o aborto na América Latina e os estudos de mulher. Revista Brasileira de Estudos de População 1989; 6:35-60.

78. Duarte GA, Osis MJD. A pesquisa sobre aborto nas ciências da saúde. In: Seminário Novas Legalidades e Democratização da Vida Social: Família, Sexualidade e Aborto. Recife: Centro Latino-Americano em Sexualidade e Direitos Humanos; 2005.

79. Zamudio-Càrdenas L, Rubiano N. Primer Encuentro de Investigadores sobre Aborto Inducido en América Latina y el Caribe, 1994: conclusiones y recomendaciones. Santafé de Bogotá: Universidad Externado de Colombia; 1995.

80. Guillaume A, Lerner S. El aborto en América Latina y el Caribe: una revisión de la literatura de los años 1990 a 2005. Paris: Centre Population et Développement; 2006.

81. Fonseca W, Misago C, Fernandes L, Correia LL, Silveira $\mathrm{D}$. Uso da aspiração manual a vácuo na redução do custo e duração de internamentos por aborto incompleto em Fortaleza, CE, Brasil. Rev Saúde Pública 1997; 31:472-8.

82. Fischer RC, Stanford JB, Jameson P, Dwitt MJ. Exploring the concepts of intended, planned, and wanted pregnancy. J Fam Pract 1999; 48:117-22.
Recebido em 06/Jan/2009
Versão final reapresentada em 10/Abr/2009
Aprovado em 20/Abr/2009 